\title{
Real-World Bleeding and Ischemic Events in Asian Patients on P2Y12-Inhibitors After Percutaneous Coronary Intervention: A National Claims Data Analysis
}

Yonggu Lee $\cdot$ Young-Hyo Lim $\cdot$ Yongwhi Park $\cdot$ Jinho Shin

Received: September 11, 2020 / Accepted: October 6, 2020 / Published online: November 11, 2020

(C) The Author(s) 2020

\begin{abstract}
Introduction: The safety and effectiveness of potent P2Y12 inhibitors in East Asians have been questioned because of the higher bleeding tendency and lower thrombotic risk in this population. We comparatively evaluated the safety, effectiveness and treatment persistence of the dual antiplatelet therapies (DAPT) with clopidogrel (CDAPT), ticagrelor (TDAPT) and prasugrel (PDAPT) after percutaneous coronary intervention (PCI) in the Korean population.

Methods: A retrospective cohort study was conducted using Korean National Health Insurance claims data. In 57,197 patients
\end{abstract}

Yonggu Lee and Young-Hyo Lim are co-first authors.

Digital Features To view digital features for this article go to https://doi.org/10.6084/m9.figshare.13050788.

Electronic Supplementary Material The online version of this article (https://doi.org/10.1007/s12325020-01526-4) contains supplementary material, which is available to authorized users.

Y. Lee · Y.-H. Lim · J. Shin $(\bowtie)$

Division of Cardiology, College of Medicine,

Hanyang University College of Medicine, Seoul,

Republic of Korea

e-mail: jhs2003@hanyang.ac.kr

Y. Park

Department of Internal Medicine, Gyeongsang

National University School of Medicine and

Gyeongsang National University Changwon

Hospital, Changwon, Republic of Korea treated with DAPT after PCI, the risk of bleeding events, risk of major adverse cardiac and cerebral events (MACCE: a composite of all-cause death, myocardial infarction $[\mathrm{MI}]$, stroke and revascularization), risk of net adverse clinical events (NACE) and persistence and adherence rates were assessed with stabilized inverse probability of treatment weighting.

Results: TDAPT was associated with higher risks of bleeding (1 year: hazard ratio [HR], 1.37; 95\% confidence interval [CI] 1.28-1.46; prolonged: HR 1.39, 95\% CI 1.31-1.47), MACCE (1 year: HR 1.10, 95\% CI 1.03-1.18; prolonged: HR $1.24,95 \%$ CI 1.16-1.31) and NACE (1 year: HR 1.23, 95\% CI 1.18-1.29; prolonged: HR 1.31, 95\% CI 1.25-1.36) than CDAPT both at 1 year and in the prolonged periods, whereas there were no significant differences between PDAPT and CDAPT. Similar results were also observed in a subgroup analysis of patients with baseline MI. CDAPT was associated with higher persistence and adherence rates than TDAPT and PDAPT.

Conclusions: CDAPT was associated with clinical outcomes that were more favorable than those in TDAPT and comparable to those in PDAPT and drug persistence and adherence that were higher than in TDAPT or PDAPT. Clopidogrel may remain a viable first option for postPCI DAPT in East Asian patients with a low thrombotic risk and a high bleeding tendency. 
Keywords: Acute coronary syndrome; Clopidogrel; East Asian patients; Percutaneous coronary intervention; Platelet aggregation inhibitors; Prasugrel; Purinergic P2Y receptor antagonists; Ticagrelor

\section{Key Summary Points}

Why carry out this study?

Recent European guidelines recommended potent P2Y12 inhibitors including ticagrelor and prasugrel over clopidogrel in acute coronary syndromes because of their rapid onset of action and strong antiplatelet activity.

The safety and effectiveness of potent P2Y12 inhibitors have been questioned in East-Asian patients with a low thrombotic risk and a high bleeding tendency.

\section{What was learned from the study?}

Clopidogrel-based dual antiplatelet therapy (DAPT) was associated with a lower risk of bleeding events, major adverse cardiocerebrovascular events and net adverse cardiovascular events than ticagrelor-based DAPT and showed a comparable risk of those events with prasugrel-based DAPT in East-Asian patients undergoing PCI.

The higher adherence to clopidogrel-based DAPT may explain the better ischemic event-related outcomes in patients on clopidogrel-based DAPT and support clopidogrel as a viable first-choice P2Y12 inhibitor for DAPT after PCI.

\section{DIGITAL FEATURES}

This article is published with digital features to facilitate understanding of the article. You can access the digital features on the article's associated Figshare page. To view digital features for this article go to https://doi.org/10.6084/m9. figshare.13050788

\section{INTRODUCTION}

Dual antiplatelet therapy (DAPT) with aspirin and a P2Y12 inhibitor is the cornerstone of preventing thrombotic events in patients undergoing percutaneous coronary intervention (PCI) [1, 2]. Although 12-month clopidogrel-based DAPT (CDAPT) has been the standard therapy after PCI since 2001 [3], recent European guidelines recommended potent P2Y12 inhibitors, including ticagrelor and prasugrel, over clopidogrel in patients with acute coronary syndrome (ACS) because of the rapid onset of action and strong antiplatelet activity of the potent P2Y12 inhibitors [4-10]. Conversely, potent P2Y12 inhibitors were reported to be associated with a higher risk of bleeding than clopidogrel, which could be even higher in EastAsian patients including Koreans [11-15].

According to the PLATO study, ticagrelor significantly reduced the risk of death from vascular causes, myocardial infarction (MI) or stroke compared with clopidogrel. However, the study also showed that the risk of postprocedural major bleeding was higher with ticagrelor than with clopidogrel $[11,15]$. The PHILO study also reported that the rates of major bleeding and composite events of MI, stroke and death from vascular causes were numerically higher in the ticagrelor group than in the clopidogrel group, although the difference was not significant [16]. Recent real-world studies also reported conflicting results on the comparative risks of bleeding and ischemic events between potent P2Y12 inhibitors and clopidogrel [17-19]. Moreover, only a few studies have compared the long-term safety and efficacy of different P2Y12 inhibitors in a real-world population undergoing PCI.

In this study, we aimed to evaluate the comparative safety and effectiveness of both standard (12 months) and prolonged DAPT (up to 48 months) among patients prescribed CDAPT, prasugrel-based DAPT (PDAPT) and ticagrelor-based DAPT (TDAPT) after PCI in real- 
world clinical settings using Korean populationbased claims data.

\section{METHODS}

\section{Data Source}

This study used national claims data from the Health Insurance Review and Assessment (HIRA) database in Korea. South Korea has a single-payer, universal and compulsory health coverage system that covers approximately 98\% of the entire South Korean population. The HIRA database contains demographic and medical claims information, including diagnosis, procedure and prescription records from inand outpatient services as well as complete follow-up data from routine clinical practice as determined by physicians. Therefore, the HIRA database represents the current clinical practices in the 50 million South Korean population [20].

The diagnoses were recorded using the Korean Classification of Disease (KCD-7) codes, which are modifications of the International Classification of Disease (ICD-10) codes. This study was based on the Korean National Health Insurance Claims Data and all records containing personal information were deidentified to protect the privacy of the patients. The permissions to access the database were obtained from the HIRA review committee (M20180511176) and exempted from review by the Institutional Review Board of Hanyang University Hospital (HYUH 2018-04-025).

\section{Study Population and Definitions}

We selected patients who underwent PCI from January 1, 2014, to December 31, 2016, and received DAPT including aspirin and one of the following P2Y12 inhibitors at the first outpatient visit after the index PCI: clopidogrel, ticagrelor or prasugrel. Patients who underwent PCI or coronary artery bypass graft (CABG) surgery or those who were prescribed P2Y12 inhibitors within 24 months before the index PCI were excluded to enroll only those who were newly treated with DAPT after PCI. We also excluded patients who had severe liver or end-stage kidney disease or were prescribed oral anticoagulants or any other oral antithrombotic agents except aspirin and P2Y12 inhibitors throughout the study period. The details of the patient selection process are presented in Fig. 1 and Table S1 in the Supplementary Material. The selected patients were followed up for a minimum of 12 months and a maximum of 48 months to evaluate the safety and effectiveness of standard and prolonged DAPT. The follow-up ended when the index DAPT was discontinued, the study endpoint occurred or the study period ended, and the elapsed time until the earliest end point occurred was considered the follow-up duration. The index DAPT was considered to be discontinued when the prescription of the index DAPT was stopped or switched to the other types of DAPT for $>30$ days.

Because the National Health Insurance reimbursement policies during the index period did not indicate whether MI was transmural, the KCD-7 codes could not represent the types of MI accurately in the HIRA database. Thus, the index events for PCI were categorized into two types, MI and non-MI, using the I21-I23 codes. Severe liver disease was defined as K74.X and end-stage kidney disease was defined as N18.5 in the KCD-7 codes.

\section{Study Outcomes}

Safety was assessed on the basis of cerebral, gastrointestinal, respiratory, urogenital and unspecified bleeding rates at 12 months and in the prolonged period (up to 48 months) after the index date. The bleeding definitions were based on the GUSTO (Global Utilization of Streptokinase and Tissue plasminogen activator for Occluded coronary arteries) criteria [21], but were determined by the diagnosis codes. Severe bleeding was defined as having a transfusion code or an admission with primary or secondary diagnosis of bleeding (Table S2 in the Supplementary Material). 


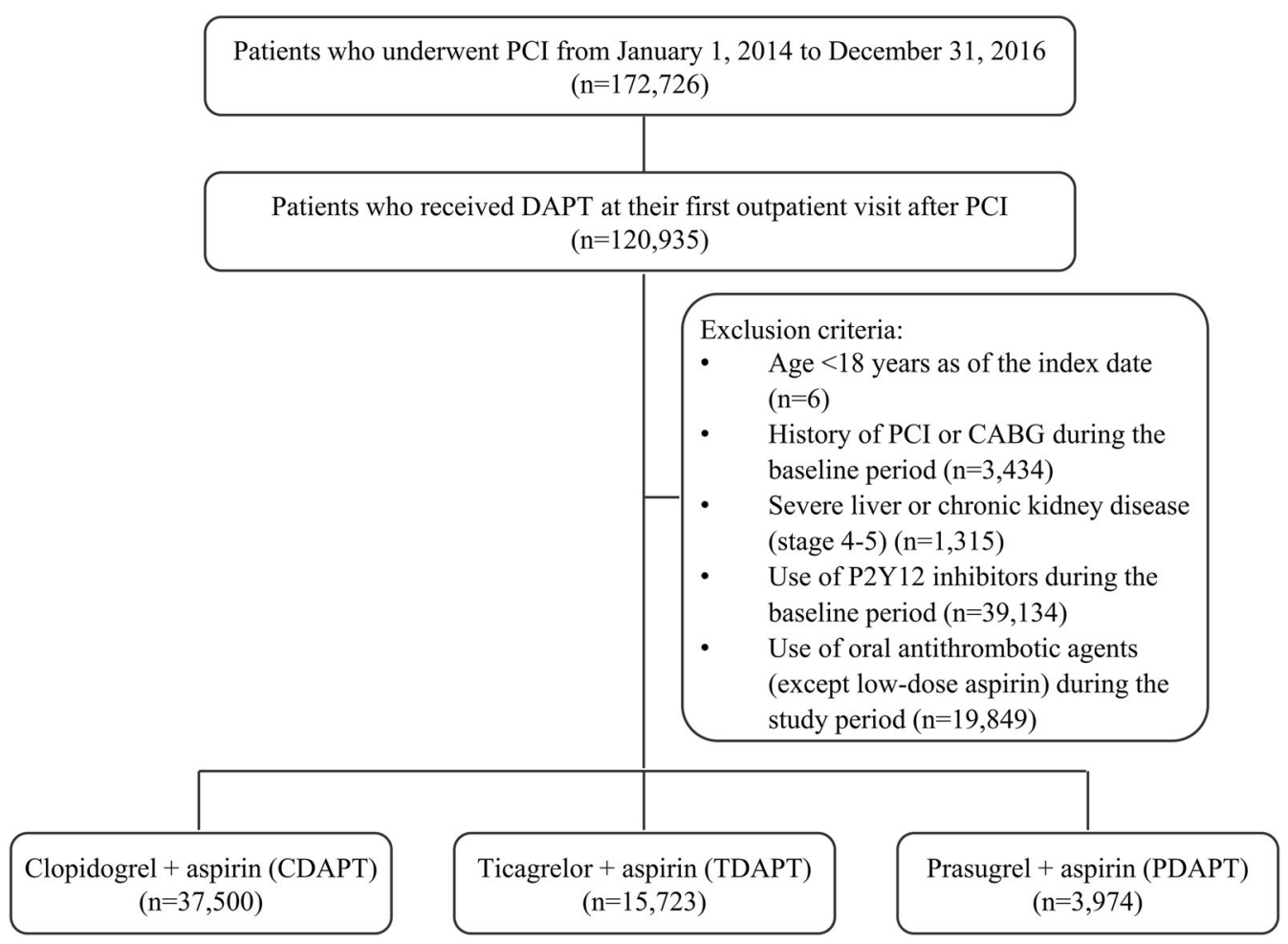

Fig. 1 Flowchart of study population enrollment. $D A P T$ dual antiplatelet therapy, $P C I$ percutaneous coronary intervention, $C A B G$ coronary artery bypass graft

A major adverse cardiac and cerebral event (MACCE) was defined as a composite event of all-cause death, stroke, MI and revascularization. All-cause death was defined using the diagnosis code or a treatment result code indicating death. Stroke was defined as an occurrence of hospital admission with stroke diagnosis codes. As a type of outcome, MI was defined as an occurrence of hospital admission or revascularization with the MI diagnosis codes. Revascularization was defined using procedure codes for PCI or CABG. A net adverse clinical event (NACE) was defined as a composite of bleeding and MACCE.

Medication persistence was measured as the proportion of patients persistent on the index DAPT and was further assessed to determine "stop," "restart" and "switch" based on subsequent prescription after discontinuation. Patients were considered persistent if they renewed their index DAPT prescription within a defined grace period of 30 days from the end of the previous prescription. Adherence was measured using the medication possession ratio (MPR), and patients with $\mathrm{MPR} \geq 80 \%$ were considered adherent [22].

\section{Statistical Methods}

Continuous variables were expressed as the mean \pm standard deviation, and categorical variables were expressed as the number (\%). Continuous variables were compared with analysis of variance (ANOVA), and categorical variables were compared with the chi-square test. Stabilized inverse probability of treatment weighting (sIPTW) using propensity scores was conducted to create comparability among the three study groups. Propensity scores were estimated using a logistic regression model including the following variables as covariates: age, sex, comorbidities, modified Charlson comorbidity index (mCCI), concomitant medications within 60 days before and after the index date, history of bleeding, number of stents implanted at the index PCI, MI diagnosis at the index PCI, 
history of low-dose aspirin use, insurance type and index year. The absolute standardized mean differences (ASDs) were compared to evaluate the balance between the study groups. An ASD of $<0.1$ was considered an indicator of a wellbalanced covariate.

Crude and weighted incidence rates were calculated as the number of outcome events per 1000 person-years at risk and presented with 95\% confidence intervals. The risk of outcomes among the study groups was compared using survival analysis with weighted Kaplan-Meier curves with sIPTW and multivariable weighted Cox proportional hazard regression models with sIPTW. Survival curves were compared using the log-rank test. Patients receiving CDAPT were used as a reference group in the Cox regression analysis.

Subgroup analyses focusing on patients diagnosed with MI at the index PCI were also conducted to investigate potential bias as the treatment selection and strategy could be different between patients with MI at the index PCI (baseline MI) and those who were not diagnosed with MI at the index PCI.

All statistical tests were two-sided with a significance level of 0.05 . The statistical analysis was performed using SAS 9.3 (SAS Institute, Cary, NC, USA).

\section{RESULTS}

\section{Baseline Characteristics}

Among the included 57,197 patients, 37,500 $(65.6 \%)$ were treated with CDAPT, 15,723 (27.5\%) with TDAPT and 3974 (6.9\%) with PDAPT (Fig. 1). Before sIPTW adjustment, there were substantial differences among the CDAPT, TDAPT and PDAPT groups. The CDAPT group was older, had higher mCCI scores and had higher proportions of women and patients with a bleeding history than the potent P2Y12 inhibitor groups, whereas the potent P2Y12 inhibitor groups had higher stent counts at the index PCI and higher proportions of patients with MI (Table 1; Table S3 in the Supplementary Material). After sIPTW, there was no significant difference in the baseline characteristics among the study groups (Table 1; Figure S1 in the supplementary material). The median follow-up time was 366 days (interquartile range [IQR], 259-532 days).

\section{Risk of Bleeding Event}

The crude incidence rate of any bleeding event was the highest in the TDAPT group and the lowest in the PDAPT group, both at 1 year and in the prolonged period. After sIPTW adjustment, TDAPT was associated with a higher risk of any bleeding event and bleeding with transfusion at 1 year and in the prolonged period than CDAPT, whereas there were no significant differences between PDAPT and CDAPT, both at 1 year and in the prolonged period (Table 2; Fig. 2).

Similar to the results observed in the entire study population, in the baseline MI subgroup, the adjusted risk of any bleeding was higher in the TDAPT group than in the CDAPT group, whereas there was no significant difference between the PDAPT and CDAPT groups (Table S4 in the Supplementary Material).

\section{Risk of MACCE}

The crude incidence rate of MACCE was the highest in the TDAPT group and the lowest in the CDAPT group both at 1 year and in the prolonged period. After sIPTW adjustment, the risk of MACCE was significantly higher in the TDAPT group than in the CDAPT group, both at 1 year and in the prolonged period, whereas there was no significant difference between the PDAPT and CDAPT groups (Table 2; Fig. 2).

Among the components of MACCE, the risks of all-cause death and stroke were significantly lower in the PDAPT group than in the CDAPT group, both at 1 year and in the prolonged period, whereas there was no significant difference between TDAPT and CDAPT. At 1 year, TDAPT was associated with a higher risk of MI than CDAPT, whereas there was no difference in the MI incidence between PDAPT and CDAPT. During the prolonged period, both TDAPT and PDAPT were associated with higher risks of MI 
Table 1 Baseline characteristics after stabilized inverse probability of treatment weighting adjustment

\begin{tabular}{|c|c|c|c|c|c|c|}
\hline & \multirow{2}{*}{$\begin{array}{l}\text { CDAPT } \\
(n=37,519)\end{array}$} & \multirow{2}{*}{$\begin{array}{l}\text { TDAPT } \\
(n=15,797)\end{array}$} & \multirow{2}{*}{$\begin{array}{l}\text { PDAPT } \\
(n=3960)\end{array}$} & \multicolumn{3}{|l|}{ ASD } \\
\hline & & & & $\begin{array}{l}\text { CDAPT vs. } \\
\text { TDAPT }\end{array}$ & $\begin{array}{l}\text { CDAPT vs. } \\
\text { PDAPT }\end{array}$ & $\begin{array}{l}\text { TDAPT vs. } \\
\text { PDAPT }\end{array}$ \\
\hline Age, years & $62.5 \pm 12.1$ & $62.7 \pm 12.1$ & $62.3 \pm 11.6$ & 0.00 & 0.00 & 0.00 \\
\hline 18 to $<45$ & $2508(6.7)$ & $1057(6.7)$ & $262(6.6)$ & 0.00 & 0.00 & 0.00 \\
\hline 45 to $<55$ & $7569(20.2)$ & $3159(20.0)$ & $812(20.5)$ & 0.00 & -0.01 & -0.01 \\
\hline 55 to $<65$ & $11,131(29.7)$ & $4631(29.3)$ & $1175(29.7)$ & 0.01 & 0.00 & -0.01 \\
\hline 65 to $<75$ & $9397(25.0)$ & $3923(24.8)$ & $1012(25.5)$ & 0.00 & -0.01 & -0.02 \\
\hline $75+$ & $6914(18.4)$ & $3028(19.2)$ & $700(17.7)$ & -0.02 & 0.02 & 0.04 \\
\hline Men & $27,659(73.7)$ & $11,587(73.4)$ & $2894(73.1)$ & 0.01 & 0.01 & 0.01 \\
\hline $\begin{array}{l}\text { Charlson } \\
\text { Comorbidity } \\
\text { Index }\end{array}$ & $1.58 \pm 1.77$ & $1.58 \pm 1.77$ & $1.57 \pm 1.78$ & 0.00 & 0.00 & 0.00 \\
\hline 0,1 & $21,390(57.0)$ & $9000(57.0)$ & $2272(57.4)$ & 0.00 & -0.01 & -0.01 \\
\hline 2 & $6131(16.3)$ & $2567(16.3)$ & $624(15.8)$ & 0.00 & 0.02 & 0.01 \\
\hline 3 & $5324(14.2)$ & $2230(14.1)$ & $596(15.1)$ & 0.00 & -0.02 & -0.03 \\
\hline $4+$ & $4674(12.5)$ & $1999(12.7)$ & $468(11.8)$ & -0.01 & 0.02 & 0.03 \\
\hline \multicolumn{7}{|l|}{ Comorbidity } \\
\hline Hypertension & $18,493(49.3)$ & 7787 (49.3) & $1950(49.2)$ & 0.00 & 0.00 & 0.00 \\
\hline Hyperlipidemia & $11,736(31.3)$ & $4862(30.8)$ & $1270(32.1)$ & 0.01 & -0.02 & -0.03 \\
\hline Diabetes & $10,077(26.9)$ & $4241(26.8)$ & $1071(27.1)$ & 0.00 & 0.00 & 0.00 \\
\hline CKD stage $1-3$ & $188(0.5)$ & $79(0.5)$ & $14(0.4)$ & 0.00 & 0.02 & 0.02 \\
\hline CKD stage $4-5$ & $126(0.3)$ & $52(0.3)$ & $8(0.2)$ & 0.00 & 0.02 & 0.02 \\
\hline Stroke & $792(2.1)$ & $334(2.1)$ & $95(2.4)$ & 0.00 & -0.02 & -0.02 \\
\hline Neoplasm & $172(0.5)$ & $68(0.4)$ & $16(0.4)$ & 0.00 & 0.01 & 0.01 \\
\hline Number of stents & $1.42 \pm 0.82$ & $1.43 \pm 0.80$ & $1.44 \pm 0.79$ & 0.00 & 0.00 & 0.00 \\
\hline 0,1 & $25,089(66.9)$ & $10,481(66.3)$ & $2624(66.3)$ & 0.01 & 0.01 & 0.00 \\
\hline 2 & 8577 (22.9) & 3664 (23.2) & $936(23.6)$ & -0.01 & -0.02 & -0.01 \\
\hline $3+$ & $3853(10.3)$ & $1652(10.5)$ & $400(10.1)$ & -0.01 & 0.01 & 0.01 \\
\hline $\begin{array}{l}\text { ACE inhibitor and } \\
\text { ARBs }\end{array}$ & $25,867(68.9)$ & $10,927(69.2)$ & $2753(69.5)$ & 0.00 & -0.01 & -0.01 \\
\hline$\beta$-blockers & $25,775(68.7)$ & $10,799(68.4)$ & $2664(67.3)$ & 0.01 & 0.03 & 0.02 \\
\hline $\mathrm{CCBs}$ & $8822(23.5)$ & 3778 (23.9) & $968(24.4)$ & -0.01 & -0.02 & -0.01 \\
\hline Statins & $34,389(91.7)$ & $14,418(91.3)$ & $3590(90.7)$ & 0.01 & 0.04 & 0.02 \\
\hline Loop diuretics & 4809 (12.8) & $2076(13.1)$ & $510(12.9)$ & -0.01 & 0.00 & 0.01 \\
\hline
\end{tabular}


Table 1 continued

\begin{tabular}{|c|c|c|c|c|c|c|}
\hline & \multirow{2}{*}{$\begin{array}{l}\text { CDAPT } \\
(n=37,519)\end{array}$} & \multirow{2}{*}{$\begin{array}{l}\text { TDAPT } \\
(n=15,797)\end{array}$} & \multirow{2}{*}{$\begin{array}{l}\text { PDAPT } \\
(n=3960)\end{array}$} & \multicolumn{3}{|l|}{ ASD } \\
\hline & & & & $\begin{array}{l}\text { CDAPT vs. } \\
\text { TDAPT }\end{array}$ & $\begin{array}{l}\text { CDAPT vs. } \\
\text { PDAPT }\end{array}$ & $\begin{array}{l}\text { TDAPT vs. } \\
\text { PDAPT }\end{array}$ \\
\hline $\begin{array}{l}\text { Diabetes } \\
\text { medication }\end{array}$ & $9031(24.1)$ & $3803(24.1)$ & $962(24.3)$ & 0.00 & -0.01 & 0.00 \\
\hline NSAIDs & $8862(23.6)$ & $3742(23.7)$ & $934(23.6)$ & 0.00 & 0.00 & 0.00 \\
\hline PPIs & $11,827(31.5)$ & $5011(31.7)$ & $1273(32.1)$ & 0.00 & -0.01 & -0.01 \\
\hline $\begin{array}{l}\text { ACE inhibitor and } \\
\text { ARBs }\end{array}$ & $24,153(64.4)$ & $10,153(64.3)$ & $2583(65.2)$ & 0.00 & -0.02 & -0.02 \\
\hline$\beta$-blockers & $26,119(69.6)$ & $11,334(71.8)$ & $2700(68.2)$ & -0.05 & 0.03 & 0.08 \\
\hline $\mathrm{CCBs}$ & $6058(16.1)$ & $2429(15.4)$ & $590(14.9)$ & 0.02 & 0.03 & 0.01 \\
\hline Statins & $33,404(89.0)$ & $14,080(89.1)$ & $3416(86.3)$ & 0.00 & 0.08 & 0.09 \\
\hline Loop diuretics & $4732(12.6)$ & $2127(13.5)$ & $501(12.6)$ & -0.03 & 0.00 & 0.02 \\
\hline $\begin{array}{l}\text { Diabetes } \\
\text { medication }\end{array}$ & $9232(24.6)$ & $3931(24.9)$ & $994(25.1)$ & -0.01 & -0.01 & -0.01 \\
\hline NSAIDs & $7038(18.8)$ & $2828(17.9)$ & $709(17.9)$ & 0.02 & 0.02 & 0.00 \\
\hline PPIs & $10,350(27.6)$ & $4456(28.2)$ & $1158(29.2)$ & -0.01 & -0.04 & -0.02 \\
\hline MI at the index PCI & $19,794(52.8)$ & $8314(52.6)$ & $2046(51.7)$ & 0.00 & 0.02 & 0.02 \\
\hline History of bleeding & $11,009(29.3)$ & $4630(29.3)$ & $1203(30.4)$ & 0.00 & -0.02 & -0.02 \\
\hline $\begin{array}{l}\text { Prior low-dose } \\
\text { aspirin usage }\end{array}$ & $12,285(32.7)$ & $5102(32.3)$ & $1314(33.2)$ & 0.01 & -0.01 & -0.02 \\
\hline \multicolumn{7}{|l|}{ Index year } \\
\hline 2014 & $12,017(32.0)$ & $5240(33.2)$ & $1146(28.9)$ & -0.02 & 0.07 & 0.09 \\
\hline 2015 & $12,210(32.5)$ & $5095(32.3)$ & $1285(32.4)$ & 0.01 & 0.00 & 0.00 \\
\hline 2016 & $13,293(35.4)$ & $5461(34.6)$ & $1530(38.6)$ & 0.02 & -0.07 & -0.08 \\
\hline \multicolumn{7}{|l|}{ Insurance type } \\
\hline Health insurance & $35,894(95.7)$ & $15,078(95.5)$ & $3769(95.2)$ & 0.01 & 0.02 & 0.01 \\
\hline Medical aid & $1625(4.3)$ & $719(4.5)$ & $192(4.8)$ & -0.01 & -0.02 & -0.01 \\
\hline \multicolumn{7}{|c|}{ Medical specialty at the index PCI } \\
\hline Internal medicine & $37,319(99.5)$ & $15,686(99.3)$ & $3953(99.8)$ & 0.02 & -0.06 & -0.08 \\
\hline Others & $200(0.5)$ & $110(0.7)$ & $8(0.2)$ & -0.02 & 0.06 & 0.08 \\
\hline \multicolumn{7}{|l|}{$\begin{array}{l}\text { Hospital type at the } \\
\text { index PCI }\end{array}$} \\
\hline Tertiary hospital & $17,233(45.9)$ & $7355(46.6)$ & $1809(45.7)$ & -0.01 & 0.01 & 0.02 \\
\hline General hospital & $20,039(53.4)$ & $8345(52.8)$ & $2128(53.7)$ & 0.01 & -0.01 & -0.02 \\
\hline
\end{tabular}


Table 1 continued

\begin{tabular}{lllllll}
\hline & $\begin{array}{l}\text { CDAPT } \\
(n=37,519)\end{array}$ & $\begin{array}{l}\text { TDAPT } \\
(n=15,797)\end{array}$ & $\begin{array}{l}\text { PDAPT } \\
(n=3960)\end{array}$ & $\begin{array}{l}\text { ASD } \\
\text { CDAPT vs. } \\
\text { TDAPT }\end{array}$ & $\begin{array}{l}\text { CDAPT vs. } \\
\text { PDAPT }\end{array}$ & $\begin{array}{l}\text { TDAPT vs. } \\
\text { PDAPT }\end{array}$ \\
\hline $\begin{array}{l}\text { Hospital } \\
\begin{array}{l}\text { General } \\
\text { practitioner }\end{array}\end{array}$ & $216(0.6)$ & $85(0.5)$ & $24(0.6)$ & 0.01 & 0.00 & -0.01 \\
\hline
\end{tabular}

$C D A P T$ clopidogrel-based dual antiplatelet therapy, TDAPT ticagrelor-based dual antiplatelet therapy, $P D A P T$ prasugrelbased dual antiplatelet therapy, $A S D$ absolute standardized mean difference, $C K D$ chronic kidney disease, $P C I$ percutaneous coronary intervention, $A C E$ angiotensin-converting enzyme, $A R B s$ angiotensin II receptor blockers, $C C B s$ calcium channel blockers, NSAIDs nonsteroidal anti-inflammatory drugs, PPIs proton-pump inhibitors, $M I$ myocardial infarction The inverse probability of treatment weight was calculated using covariates such as age, sex, comorbidity, number of stents implanted at the index PCI, history of bleeding, concomitant drugs, hospital type at the index PCI, index year and insurance type. Values are presented as $n(\%)$

and revascularization than CDAPT (Table 2; Figure S2 in the Supplementary Material).

Similarly, the adjusted risk of MACCE was significantly higher in the TDAPT group than in the CDAPT group among the baseline MI patients, whereas there was no significant difference between the PDAPT and CDAPT groups in the baseline MI subgroup (Table S4 in the Supplementary Material).

\section{Risk of NACE}

The crude incidence rate of NACE was the highest in the TDAPT group and the lowest in the CDAPT group during the study period. After sIPTW adjustment, TDAPT was associated with an increased risk of NACE compared with CDAPT both at 1 year and in the prolonged period, whereas there was no significant difference in the incidence of NACE between PDAPT and CDAPT (Table 2; Fig. 2).

In the MI subgroup, the sIPTW-adjusted NACE also yielded results similar to those observed in the entire study population both at 1 year and in the prolonged period (Table S4 in the Supplementary Material).

\section{Medication Persistence and Adherence}

Of the 57,197 patients, $55 \%$ were persistent on their index DAPT until the end of the 1-year period and $25.7 \%$ were persistent until the end of the prolonged period. On average, $91.3 \%$ of the total study population was adherent with an MPR $\geq 0.8$ both at 1 year and in the prolonged period.

The persistence and adherence rates with MPR $\geq 0.8$ were significantly higher in the CDAPT group than in the TDAPT and PDAPT groups for 1 year and the prolonged period. Of patients in the CDAPT group, 34.9\% remained on CDAPT until the end of the prolonged period, whereas only $7.8 \%$ and $9.2 \%$ remained on TDAPT and PDAPT, respectively. Of those who switched their index DAPT within 12 months after PCI, $93.7 \%$ and $97.4 \%$ patients switched to CDAPT from TDAPT or PDAPT, respectively (Table 3; Figure S3 in the Supplementary Material).

\section{DISCUSSION}

Our study found that CDAPT was associated with a lower risk of bleeding events, MACCE and NACE than TDAPT, whereas the risks were comparable between CDAPT and PDAPT among Koreans in real-world settings. Patients on CDAPT were more likely to persist on the index DAPT, whereas those on TDAPT or PDAPT tended to change their index DAPT to CDAPT.

The clinical characteristics of the patients seemed to be important to physicians when 
Table 2 Incidence rates and hazard ratios of bleeding, MACCE and NACE

\begin{tabular}{|c|c|c|c|c|c|c|c|}
\hline & \multicolumn{3}{|c|}{$\begin{array}{l}\text { Crude incidence rate }(/ 1000 \text { person- } \\
\text { years })\end{array}$} & \multicolumn{4}{|c|}{ sIPTW weighted hazard ratio } \\
\hline & \multirow{2}{*}{$\begin{array}{l}\text { CDAPT } \\
(n=37,500)\end{array}$} & \multirow{2}{*}{$\begin{array}{l}\text { TDAPT } \\
(n=15,723)\end{array}$} & \multirow{2}{*}{$\begin{array}{l}\text { PDAPT } \\
(n=3974)\end{array}$} & \multicolumn{2}{|c|}{$\begin{array}{l}\text { TDAPT vs. CDAPT } \\
\text { CDP }\end{array}$} & \multicolumn{2}{|c|}{ PDAPT vs. CDAPT } \\
\hline & & & & $\begin{array}{l}\text { HR }(95 \% \\
\text { CI) }\end{array}$ & $p$ value & $\begin{array}{l}\text { HR }(95 \% \\
\text { CI) }\end{array}$ & $p$ value \\
\hline \multicolumn{8}{|l|}{ 1-Year follow-up } \\
\hline Any bleeding & 104.9 & 125.0 & 89.8 & $\begin{array}{l}1.37 \\
(1.28-1.46)\end{array}$ & $<0.001$ & $\begin{array}{l}1.01 \\
\quad(0.90-1.14)\end{array}$ & 0.847 \\
\hline Cerebral bleeding & 9.6 & 6.7 & 4.4 & $\begin{array}{l}1.03 \\
(0.82-1.30)\end{array}$ & 0.805 & $\begin{array}{l}1.67 \\
(1.20-2.33)\end{array}$ & 0.002 \\
\hline $\begin{array}{l}\text { Gastro-intestinal } \\
\text { bleeding }\end{array}$ & 29.8 & 34.3 & 25.7 & $\begin{array}{l}1.29 \\
(1.14-1.45)\end{array}$ & $<0.001$ & $\begin{array}{l}0.78 \\
\quad(0.61-0.99)\end{array}$ & 0.043 \\
\hline $\begin{array}{l}\text { Respiratory track } \\
\text { bleeding }\end{array}$ & 24.2 & 49.4 & 36.3 & $\begin{array}{l}2.28 \\
\quad(2.04-2.54)\end{array}$ & $<0.001$ & $\begin{array}{l}1.42 \\
(1.16-1.75)\end{array}$ & 0.001 \\
\hline $\begin{array}{l}\text { Urogenital } \\
\text { bleeding }\end{array}$ & 18.6 & 18.2 & 13.1 & $\begin{array}{l}1.02 \\
(0.86-1.20)\end{array}$ & 0.845 & $\begin{array}{l}0.71 \\
\quad(0.52-0.99)\end{array}$ & 0.040 \\
\hline $\begin{array}{l}\text { Unspecified } \\
\text { bleeding }\end{array}$ & 26.5 & 20.0 & 12.8 & $\begin{array}{l}0.95 \\
\quad(0.83-1.10)\end{array}$ & 0.516 & $\begin{array}{l}0.82 \\
\quad(0.63-1.06)\end{array}$ & 0.134 \\
\hline $\begin{array}{l}\text { Bleeding with } \\
\text { admission }\end{array}$ & 21.9 & 27.8 & 18.4 & $\begin{array}{l}1.49 \\
(1.31-1.71)\end{array}$ & $<0.001$ & $\begin{array}{l}0.82 \\
\quad(0.62-1.09)\end{array}$ & 0.171 \\
\hline $\begin{array}{l}\text { Bleeding with } \\
\text { transfusion }\end{array}$ & 10.5 & 15.5 & 9.0 & $\begin{array}{l}1.95 \\
(1.63-2.33)\end{array}$ & $<0.001$ & $\begin{array}{l}0.82 \\
\quad(0.54-1.25)\end{array}$ & 0.353 \\
\hline $\begin{array}{l}\text { Bleeding with } \\
\text { transfusion } \geq 2 \\
\text { packs }\end{array}$ & 4.1 & 6.5 & 5.6 & $\begin{array}{l}2.46 \\
\quad(1.88-3.20)\end{array}$ & $<0.001$ & $\begin{array}{l}1.38 \\
\quad(0.80-2.35)\end{array}$ & 0.245 \\
\hline MACCE & 98.2 & 129.1 & 115.7 & $\begin{array}{l}1.10 \\
\quad(1.03-1.18)\end{array}$ & 0.005 & $\begin{array}{l}1.01 \\
\quad(0.90-1.13)\end{array}$ & 0.918 \\
\hline All-cause death & 3.3 & 2.4 & 0.6 & $\begin{array}{l}0.91 \\
\quad(0.61-1.38)\end{array}$ & 0.666 & $\begin{array}{l}0.19 \\
\quad(0.04-0.87)\end{array}$ & 0.032 \\
\hline Stroke & 8.0 & 5.1 & 3.7 & $\begin{array}{l}0.78 \\
\quad(0.59-1.04)\end{array}$ & 0.092 & $\begin{array}{l}0.42 \\
\quad(0.21-0.80)\end{array}$ & 0.009 \\
\hline MI & 41.7 & 72.3 & 60.7 & $\begin{array}{l}1.17 \\
\quad(1.06-1.28)\end{array}$ & 0.002 & $\begin{array}{l}1.05 \\
\quad(0.89-1.24)\end{array}$ & 0.596 \\
\hline Revascularization & 45.9 & 49.4 & 50.4 & $\begin{array}{l}1.09 \\
\quad(0.99-1.21)\end{array}$ & 0.087 & $\begin{array}{l}1.09 \\
\quad(0.92-1.28)\end{array}$ & 0.340 \\
\hline NACE & 199.6 & 249.9 & 202.4 & $\begin{array}{l}1.23 \\
\quad(1.18-1.29)\end{array}$ & $<0.001$ & $\begin{array}{l}1.00 \\
\quad(0.92-1.09)\end{array}$ & 0.951 \\
\hline
\end{tabular}


Table 2 continued

\begin{tabular}{|c|c|c|c|c|c|c|c|}
\hline & \multicolumn{3}{|c|}{$\begin{array}{l}\text { Crude incidence rate }(/ 1000 \text { person- } \\
\text { years })\end{array}$} & \multicolumn{4}{|c|}{ sIPTW weighted hazard ratio } \\
\hline & \multirow{2}{*}{$\begin{array}{l}\text { CDAPT } \\
(n=37,500)\end{array}$} & \multirow{2}{*}{$\begin{array}{l}\text { TDAPT } \\
(n=15,723)\end{array}$} & \multirow{2}{*}{$\begin{array}{l}\text { PDAPT } \\
(n=3974)\end{array}$} & \multicolumn{2}{|c|}{ TDAPT vs. CDAPT } & \multicolumn{2}{|c|}{ PDAPT vs. CDAPT } \\
\hline & & & & $\begin{array}{l}\text { HR }(95 \% \\
\text { CI) }\end{array}$ & $p$ value & $\begin{array}{l}\text { HR }(95 \% \\
\text { CI) }\end{array}$ & $p$ value \\
\hline \multicolumn{8}{|l|}{ Prolonged follow-up } \\
\hline Any bleeding & 91.6 & 119.5 & 84.8 & $\begin{array}{l}1.39 \\
\quad(1.31-1.47)\end{array}$ & $<0.0001$ & $\begin{array}{l}1.04 \\
(0.93-1.16)\end{array}$ & 0.500 \\
\hline Cerebral bleeding & 7.5 & 6.6 & 4.6 & $\begin{array}{l}1.09 \\
\quad(0.88-1.36)\end{array}$ & 0.427 & $\begin{array}{l}1.67 \\
(1.22-2.29)\end{array}$ & 0.002 \\
\hline $\begin{array}{l}\text { Gastro-intestinal } \\
\text { bleeding }\end{array}$ & 27.2 & 33.0 & 25.6 & $\begin{array}{l}1.28 \\
(1.15-1.43)\end{array}$ & $<0.001$ & $\begin{array}{l}0.81 \\
\quad(0.65-1.01)\end{array}$ & 0.066 \\
\hline $\begin{array}{l}\text { Respiratory track } \\
\text { bleeding }\end{array}$ & 21.0 & 45.2 & 33.0 & $\begin{array}{l}2.19 \\
\quad(1.97-2.42)\end{array}$ & $<0.001$ & $\begin{array}{l}1.47 \\
\quad(1.21-1.77)\end{array}$ & $<0.001$ \\
\hline $\begin{array}{l}\text { Urogenital } \\
\text { bleeding }\end{array}$ & 16.9 & 17.7 & 12.5 & $\begin{array}{l}1.05 \\
\quad(0.90-1.21)\end{array}$ & 0.569 & $\begin{array}{l}0.77 \\
\quad(0.58-1.03)\end{array}$ & 0.078 \\
\hline $\begin{array}{l}\text { Unspecified } \\
\text { bleeding }\end{array}$ & 22.5 & 20.1 & 13.0 & $\begin{array}{l}1.08 \\
(0.95-1.23)\end{array}$ & 0.257 & $\begin{array}{l}0.83 \\
\quad(0.65-1.07)\end{array}$ & 0.147 \\
\hline $\begin{array}{l}\text { Bleeding with } \\
\text { admission }\end{array}$ & 21.0 & 27.6 & 19.6 & $\begin{array}{l}1.51 \\
\quad(1.34-1.71)\end{array}$ & $<0.001$ & $\begin{array}{l}0.97 \\
\quad(0.76-1.24)\end{array}$ & 0.810 \\
\hline $\begin{array}{l}\text { Bleeding with } \\
\text { transfusion }\end{array}$ & 10.2 & 15.4 & 9.1 & $\begin{array}{l}1.93 \\
\quad(1.64-2.27)\end{array}$ & $<0.001$ & $\begin{array}{l}0.85 \\
\quad(0.59-1.24)\end{array}$ & 0.408 \\
\hline $\begin{array}{l}\text { Bleeding with } \\
\text { transfusion } \geq 2 \\
\text { packs }\end{array}$ & 4.0 & 6.4 & 5.3 & $\begin{array}{l}2.33 \\
\quad(1.83-2.96)\end{array}$ & $<0.001$ & $\begin{array}{l}1.40 \\
\quad(0.87-2.25)\end{array}$ & 0.165 \\
\hline MACCE & 88.3 & 140.4 & 124.5 & $\begin{array}{l}1.24 \\
\quad(1.16-1.31)\end{array}$ & $<0.001$ & $\begin{array}{l}1.09 \\
\quad(0.98-1.21)\end{array}$ & 0.096 \\
\hline All-cause death & 2.9 & 2.2 & 0.8 & $\begin{array}{l}0.88 \\
\quad(0.60-1.30)\end{array}$ & 0.516 & $\begin{array}{l}0.23 \\
\quad(0.06-0.84)\end{array}$ & 0.026 \\
\hline Stroke & 6.8 & 5.8 & 3.5 & $\begin{array}{l}1.06 \\
(0.84-1.36)\end{array}$ & 0.614 & $\begin{array}{l}0.42 \\
\quad(0.22-0.79)\end{array}$ & 0.007 \\
\hline MI & 35.0 & 77.3 & 61.8 & $\begin{array}{l}1.28 \\
\quad(1.18-1.40)\end{array}$ & $<0.001$ & $\begin{array}{l}1.13 \\
\quad(0.97-1.32)\end{array}$ & 0.105 \\
\hline Revascularization & 43.3 & 54.6 & 57.2 & $\begin{array}{l}1.23 \\
\quad(1.13-1.35)\end{array}$ & $<0.001$ & $\begin{array}{l}1.17 \\
\quad(1.01-1.35)\end{array}$ & 0.042 \\
\hline
\end{tabular}


Table 2 continued

\begin{tabular}{|c|c|c|c|c|c|c|c|}
\hline & \multicolumn{3}{|c|}{$\begin{array}{l}\text { Crude incidence rate }(/ 1000 \text { person- } \\
\text { years) }\end{array}$} & \multicolumn{4}{|c|}{ sIPTW weighted hazard ratio } \\
\hline & \multirow{2}{*}{$\begin{array}{l}\text { CDAPT } \\
(n=37,500)\end{array}$} & \multirow{2}{*}{$\begin{array}{l}\text { TDAPT } \\
(n=15,723)\end{array}$} & \multirow{2}{*}{$\begin{array}{l}\text { PDAPT } \\
(n=3974)\end{array}$} & \multicolumn{2}{|c|}{ TDAPT vs. CDAPT } & \multicolumn{2}{|c|}{ PDAPT vs. CDAPT } \\
\hline & & & & $\begin{array}{l}\text { HR }(95 \% \\
\text { CI })\end{array}$ & $p$ value & $\begin{array}{l}\text { HR }(95 \% \\
\text { CI) }\end{array}$ & $p$ value \\
\hline NACE & 178.0 & 256.0 & 206.4 & $\begin{array}{l}1.31 \\
(1.25-1.36)\end{array}$ & $<0.001$ & $\begin{array}{l}1.05 \\
(0.97-1.14)\end{array}$ & 0.210 \\
\hline
\end{tabular}

$C D A P T$ clopidogrel-based dual antiplatelet therapy, TDAPT ticagrelor-based dual antiplatelet therapy, $P D A P T$ prasugrelbased dual antiplatelet therapy, MACCE major adverse cardiac or cerebral event, $N A C E$ net adverse clinical event, $M I$ myocardial infarction, $s I P T W$ stabilized inverse probability of treatment weighting, $H R$ hazard ratio, $C I$ confidence interval The inverse probability of treatment weight was calculated using covariates such as age, sex, comorbidity, number of stents implanted at the index PCI, history of bleeding, concomitant drugs, hospital type at the index PCI, index year and insurance type. The $p$-values were derived from weighted Cox proportional hazard regression with sIPTW and covariates such as age, sex, comorbidity, number of stents implemented at the index PCI, history of bleeding, concomitant drugs, index year and insurance type. The CDAPT group was used as the reference group

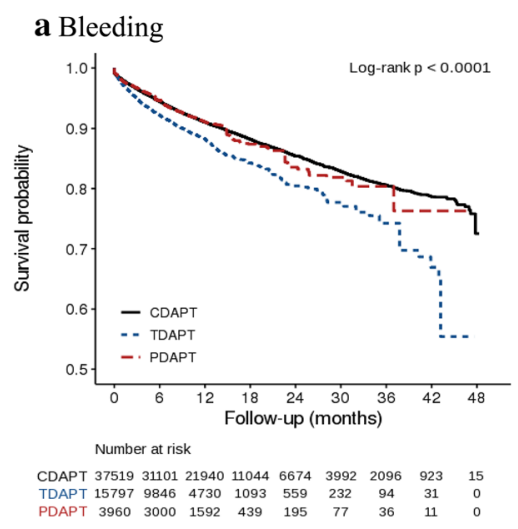

Fig. 2 Stabilized inverse probability of treatment weighting-adjusted Kaplan-Meier survival curve for bleeding, MACCE and NACE. CDAPT clopidogrel-based dual antiplatelet therapy, TDAPT ticagrelor-based dual antiplatelet therapy, PDAPT prasugrel-based dual antiplatelet therapy, MACCE major adverse cardiac or cerebral event,

selecting P2Y12 inhibitors after PCI in realworld settings. In our study, crude comparisons of baseline characteristics among the DAPT groups showed that the potent P2Y12 agent groups included younger patients with fewer comorbidities and previous bleeding histories. They were also less frequently prescribed nonsteroidal anti-inflammatory drugs than those in

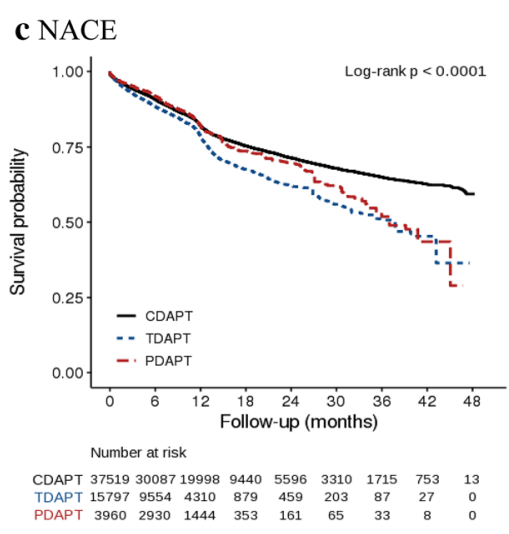

$N A C E$ net adverse clinical event. The inverse probability of treatment weight was calculated using covariates such as age, sex, comorbidity, number of stents implanted at the index percutaneous coronary intervention (PCI), history of bleeding, concomitant drugs, hospital type at the index PCI, index year and insurance type

the CDAPT group. These baseline differences suggest that physicians preferred CDAPT over DAPT with potent P2Y12 agents for patients with a higher bleeding risk. Nevertheless, actual bleeding events occurred more frequently in the TDAPT group than in the CDAPT group in our results. Moreover, although PDAPT and CDAPT exhibited similar composite bleeding risks, 
Table 3 Persistence rates, adherence rates and discontinuation patterns

\begin{tabular}{|c|c|c|c|c|c|}
\hline & $\begin{array}{l}\text { All patients } \\
(n=57,197)\end{array}$ & $\begin{array}{l}\text { CDAPT } \\
(n=37,500)\end{array}$ & $\begin{array}{l}\text { TDAPT } \\
(n=15,723)\end{array}$ & $\begin{array}{l}\text { PDAPT } \\
(n=3974)\end{array}$ & $p$ value \\
\hline \multicolumn{6}{|c|}{ Persistence rate and discontinuation pattern $(n, \%)$} \\
\hline \multicolumn{6}{|l|}{ 1-year follow-up } \\
\hline Continuation & $31,507(55.1)$ & $24,567(65.5)$ & $5170(32.9)$ & $1770(44.5)$ & $<0.001$ \\
\hline Discontinuation & $25,690(44.9)$ & $12,933(34.5)$ & $10,553(67.1)$ & $2204(55.5)$ & $<0.001$ \\
\hline Restart & $5459(9.5)$ & $4328(11.5)$ & $915(5.8)$ & $216(5.4)$ & $<0.001$ \\
\hline Stop & $12,201(21.3)$ & $8,271(22.1)$ & $3142(20.0)$ & $788(19.8)$ & $<0.001$ \\
\hline Switch & $8030(14.0)$ & $334(0.9)$ & $6496(41.3)$ & $1200(30.2)$ & $<0.001$ \\
\hline to $[\mathrm{CDAPT}]$ & $7254(90.3)$ & - & $6088(93.7)$ & $1166(97.2)$ & \\
\hline to [TDAPT] & $264(3.3)$ & $230(68.9)$ & - & $34(2.8)$ & \\
\hline to $[\mathrm{PDAPT}]$ & $512(6.4)$ & $104(31.1)$ & $408(6.3)$ & - & \\
\hline \multicolumn{6}{|l|}{$\begin{array}{l}\text { Prolonged follow- } \\
\text { up }\end{array}$} \\
\hline Continuation & $14,683(25.7)$ & $13,098(34.9)$ & $1,220(7.8)$ & $365(9.2)$ & $<0.001$ \\
\hline Discontinuation & $42,514(74.3)$ & $24,402(65.1)$ & $14,503(92.2)$ & $3609(90.8)$ & $<0.001$ \\
\hline Restart & $8237(14.4)$ & $6879(18.3)$ & $1073(6.8)$ & $285(7.2)$ & $<0.001$ \\
\hline Stop & $23,014(40.2)$ & $16,998(45.3)$ & $4678(29.8)$ & $1338(33.7)$ & $<0.001$ \\
\hline Switch & $11,263(19.7)$ & $525(1.4)$ & $8752(55.7)$ & $1986(50.0)$ & $<0.001$ \\
\hline to $[\mathrm{CDAPT}]$ & $10,239(90.9)$ & & $8301(94.8)$ & $1938(97.6)$ & \\
\hline to [TDAPT] & $596(5.3)$ & $380(72.4)$ & & $48(2.4)$ & \\
\hline to $[\mathrm{PDAPT}]$ & $428(3.8)$ & $145(27.6)$ & $451(5.2)$ & & \\
\hline \multicolumn{6}{|c|}{ Adherence rate $(n, \%)$} \\
\hline \multicolumn{6}{|l|}{ 1-Year follow-up } \\
\hline $\mathrm{MPR} \geq 0.8$ & $52,246(91.3)$ & $35,378(94.3)$ & $13,199(83.9)$ & $3669(92.3)$ & $<0.001$ \\
\hline $\mathrm{MPR}<0.8$ & $4951(8.7)$ & $2122(5.7)$ & $2524(16.1)$ & $305(7.7)$ & \\
\hline \multicolumn{6}{|l|}{$\begin{array}{l}\text { Prolonged follow- } \\
\text { up }\end{array}$} \\
\hline $\mathrm{MPR} \geq 0.8$ & $52,239(91.3)$ & $35,374(94.3)$ & $13,197(83.9)$ & $3668(92.3)$ & $<0.001$ \\
\hline $\mathrm{MPR}<0.8$ & $4958(8.7)$ & $2126(5.7)$ & $2526(16.1)$ & $306(7.7)$ & \\
\hline
\end{tabular}

$C D A P T$ clopidogrel-based dual antiplatelet therapy, TDAPT ticagrelor-based dual antiplatelet therapy, $P D A P T$ prasugrelbased dual antiplatelet therapy, $M P R$ medication possession ratio

The $p$ values were derived from individual chi-square tests and analysis of variance tests 
PDAPT was more strongly associated with a higher risk of cerebral hemorrhage than CDAPT, which can be potentially more dangerous than other forms of bleeding (Table 2). It is noteworthy that clopidogrel tended to be better maintained than both ticagrelor and prasugrel in 1 year and prolonged DAPT in our study. In summary, our results may strongly support the superior hemorrhagic safety and more reliable persistence/adherence of clopidogrel than potent P2Y12 inhibitors in post-PCI DAPT among the Korean population.

The clinical characteristics in our study are consistent with those in recent studies conducted in real-world settings. A study using Austrian national health insurance data showed that among patients with established ACS, clopidogrel users were older and more frequently prescribed cardiovascular or diabetic medications than potent P2Y12 inhibitor users [17]. Blin et al. also reported, using French national health insurance data, that clopidogrel users were older and more frequently had a CCI $\geq 6$ than ticagrelor or prasugrel users, whereas acute MI as the index ACS was more frequent among potent P2Y12 inhibitor users than among clopidogrel users [23]. However, unlike our results, the risk of bleeding was not different among the three P2Y12 inhibitors, whereas the risks of death and composite ischemic events were lower in ticagrelor users than in clopidogrel users. A recent study conducted by Kim et al., using Korean HIRA claims data [18], also found that the risk of bleeding did not differ between ticagrelor and clopidogrel, which contradicted our results. These inconsistencies may have resulted from differences in study design and population characteristics. For instance, Kim et al. included only patients with acute MI whose future risk of ischemic events would be higher than those of non-MI PCI patients and excluded patients $\geq 75$ years old, who would be the most vulnerable population to the risk of bleeding but, at the same time, would be less likely to undergo repeat revascularizations when symptoms recur. In contrast, Korean Acute Myocardial Infarction Registry researchers reported that ticagrelor was associated with a higher risk of bleeding than clopidogrel during the first 6 months after PCI, similar to our results [19].

Despite the more potent antiplatelet effects of ticagrelor and prasugrel, TDAPT was associated with a higher risk of composite MACCE than CDAPT, both at 1 year and in the prolonged period up to 4 years, and PDAPT did not show its superiority in composite MACCE reduction over CDAPT. Although we had adjusted extensively for covariates associated with the risk of ischemic events and bleeding, few changes in the results were observed, which might be accounted for by several reasons. Unmeasured confounding factors such as lesion complexity, lesion locations, perioperative complications and type of procedures and stents may have influenced P2Y12 inhibitor selection by physicians while influencing the clinical outcomes after PCI. The higher risk of MACCE was mainly driven by the higher incidence of $\mathrm{MI}$ and revascularization in the potent P2Y12 inhibitor groups. Because our study design did not allow for differentiation of the scheduled PCI performed several months after the index PCI from true ischemic events, the incidence of $\mathrm{MI}$ and revascularization might be more easily overestimated in potent P2Y12 inhibitor users who would have more complex lesions and tend to have multiple procedures for a single clinical event. In fact, death, which might potentially indicate stent thrombosis, and ischemic stroke, which would solely be associated with thrombosis, occurred less frequently in the potent P2Y12 inhibitor groups.

Our results are consistent with those from the PHILO study conducted in East-Asian patients, which reported that the incidence of bleeding and cardiovascular events was higher in ticagrelor users than in clopidogrel users [16]. In comparison with clopidogrel, a recent randomized controlled trial in Korean patients with ACS also reported a higher risk of bleeding with ticagrelor but found no significant difference in cardiovascular death [24]. These observations are in line with the phenomenon referred to as the "East Asian paradox," which describes the vulnerability to bleeding and resistance to ischemic events of East Asian populations compared with Caucasians [25]. Furthermore, these observations might suggest 
that a de-escalation strategy from potent P2Y12 inhibitors to clopidogrel should be considered to improve the outcome of DAPT treatment in real-world practices.

Unlike the comparisons between TDAPT and CDAPT, there were no significant differences in safety or effectiveness between PDAPT and CDAPT. However, the risks of all-cause death and stroke were lower in the PDAPT group than in the CDAPT groups, both at 1 year and in the prolonged period. These results may be explained by the national insurance policy of the HIRA, which does not reimburse the use of prasugrel in patients aged $\geq 75$ years, those weighing $<60 \mathrm{~kg}$ or those with a history of stroke $[26,27]$. Subsequently, PDAPT was used much less frequently, compared with the other DAPTs, and the PDAPT users were younger, more frequently male and less predisposed to stroke than CDAPT users in the data. These augmented baseline differences may have resulted in a lower risk of bleeding, all-cause death and stroke in the PDAPT group. Even after robust weighting, vastly different patient characteristics would make a fair comparison difficult [23]. Similarly, no significant differences were observed between prasugrel and clopidogrel users in a French claims database study. Nevertheless, our results showed that there was still a marginally higher risk of MACCE in the PDAPT groups in the prolonged follow-up period, mostly driven by the risk of MI and revascularization.

Current guidelines recommend the continuous use of DAPT for up to 12 months after PCI to reduce the risk of ischemic recurrence $[1,2]$. Premature discontinuation of DAPT has been known to increase the risk of ischemic events [28]. According to recent real-world studies, premature discontinuation of DAPT was less frequent in patients using clopidogrel than in those using potent P2Y12 inhibitors [17, 18]. In addition, clopidogrel was associated with higher adherence than potent P2Y12 inhibitors. These high persistence and adherence rates of clopidogrel may account for the lower risk of MACCE in the CDAPT group compared to that in the TDAPT group and the lower risk of ischemic events in the CDAPT group compared to that in the PDAPT group in the prolonged follow-up period.

\section{Limitations}

Our study had several limitations. First, this study was a retrospective observational cohort study. Any association between P2Y12 inhibitors and clinical outcomes observed in the results should be interpreted with caution, as it does not directly indicate causality. Second, unlike clinical cohort studies, some important information about lesion complexity and location at index PCI, baseline laboratory findings and cardiac function was not available. Third, we defined the clinical outcomes and comorbidities using KCD-7 codes, which could harbor some information biases from voluntary/involuntary miscoding that would lead to underestimation or overestimation of the outcomes. In particular, we could not classify the severity of bleeding owing to a lack of clinical laboratory information. However, studies have shown that approximately $70 \%$ of the primary, secondary or tertiary diagnosis codes from the insurance claims data are valid $[29,30]$. The accuracy of ICD-10 codes in the diagnosis of MI and cerebrovascular disease was reported to be $>70 \%$ and $83.4 \%$, respectively $[31,32]$. Fourth, the identification of death was limited to in-hospital death owing to the nature of claims data. Therefore, the incidence of death was lower than that in a previous study using cause of death statistics from death certificates [33]. We conducted a sensitivity analysis using a proxy definition of death, which was a combination of serious conditions (defined as $\mathrm{CCI} \geq 6$ ) and blank periods (no claims $\geq 6$ months) [34]; however, the results were similar to those of analyses conducted using the current definition of death (Table S5 in the Supplementary Material). Fifth, because our study was conducted solely in Koreans, a typical East-Asian population, it is difficult to generalize our findings in ethnic groups other than East-Asian populations. Finally, the causes of P2Y12 inhibitor discontinuation were not identified owing to the nature of claims data. 


\section{CONCLUSION}

Despite the lower potency of clopidogrel in terms of antiplatelet action, the risks of bleeding, ischemic events and NACEs in CDAPT, which were lower than those in TDAPT and comparable to those in PDAPT, may still advocate for the use of clopidogrel as a viable firstchoice for DAPT after PCI in Korean patients who have a high bleeding tendency and low ischemic risk, as is typical in other East Asian populations. In addition, the use of clopidogrel is associated with better persistence and adherence to DAPT than potent P2Y12 inhibitors, and this may contribute to the lower risk of ischemic events in the CDAPT users than in TDAPT users.

\section{ACKNOWLEDGMENTS}

Funding. This study was sponsored by Sanofi-Aventis Korea. The Rapid Service and Open Access Fees were also funded by SanofiAventis Korea.

Medical Writing and Editorial Assistance. All authors acknowledge IQVIA Korea and Sanofi-Aventis Korea for their support in assisting with the study design and execution. IQVIA Korea supported medical writing, editorial assistance and statistical analysis under the author's supervision (Jung-Ae Kim, PhD/Real World Insights, IQVIA Korea, Seoul, Republic of Korea). Sanofi-Aventis Korea reviewed the study design and this manuscript for medical accuracy. Support for this assistance was funded by Sanofi-Aventis Korea.

Authorship. All named authors meet the International Committee of Medical Journal Editors (ICMJE) criteria for authorship for this article, take responsibility for the integrity of the work as a whole and have given their approval for this version to be published.

Authorship Contributions. Y.L., Y.H.L. and J.H.S. designed the study. Y.L. and Y.H.L. analyzed the data. Y.L., Y.H.L. and J.H.S. interpreted the data and wrote the manuscript. Y.W.P. contributed the interpretation of analysis results and revision of the manuscript.

Disclosures. Jinho Shin declares that he received a research grant from Sanofi-Aventis Korea. Yonggu Lee, Young-Hyo Lim and Yongwhi Park have nothing to disclose.

Compliance With Ethics Guidelines. This study was based on the Korean National Health Insurance Claims Data and all records containing personal information were de-identified to protect the privacy of the patients. The permissions to access the database were obtained from the HIRA review committee (M20180511176) and exempted from review by the Institutional Review Board of Hanyang University Hospital (HYUH 2018-04-025).

Data Availability. The datasets generated during and/or analyzed during the current study will not be publicly available until HIRA, the data owner, grants permission for access to the data.

Open Access. This article is licensed under a Creative Commons Attribution-NonCommercial 4.0 International License, which permits any non-commercial use, sharing, adaptation, distribution and reproduction in any medium or format, as long as you give appropriate credit to the original author(s) and the source, provide a link to the Creative Commons licence, and indicate if changes were made. The images or other third party material in this article are included in the article's Creative Commons licence, unless indicated otherwise in a credit line to the material. If material is not included in the article's Creative Commons licence and your intended use is not permitted by statutory regulation or exceeds the permitted use, you will need to obtain permission directly from the copyright holder. To view a copy of this licence, visit http://creativecommons.org/licenses/by$\mathrm{nc} / 4.0 /$. 


\section{REFERENCES}

1. Rosano GMC, Seferovic P. 2017 ESC guidelines focus on dual antiplatelet therapy. Eur Heart J Cardiovasc Pharmacother. 2018;4(3):131-2. https:// doi.org/10.1093/ehjcvp/pvy007.

2. Levine GN, Bates ER, Bittl JA, et al. 2016 ACC/AHA guideline focused update on duration of dual antiplatelet therapy in patients with coronary artery disease: a report of the American College of Cardiology/American Heart Association Task Force on Clinical Practice Guidelines. J Thorac Cardiovasc Surg. 2016;152(5):1243-75. https://doi.org/10. 1016/j.jacc.2016.03.513.

3. Ko DT, Krumholz HM, Tu JV, et al. Clinical outcomes of plavix and generic clopidogrel for patients hospitalized with an acute coronary syndrome. Circ Cardiovasc Qual Outcomes. 2018;11(3):e004194. https://doi.org/10.1161/CIRCOUTCOMES.117. 004194.

4. Yudi MB, Clark DJ, Farouque O, et al. Clopidogrel, prasugrel or ticagrelor in patients with acute coronary syndromes undergoing percutaneous coronary intervention. Intern Med J. 2016;46(5):559-65. https://doi.org/10.1111/imj.13041.

5. Karve AM, Seth M, Sharma M, et al. Contemporary use of ticagrelor in interventional practice (from Blue Cross Blue Shield of Michigan Cardiovascular Consortium). Am J Cardiol. 2015;115(11):1502-6. https://doi.org/10.1016/j.amjcard.2015.02.049.

6. Wijeyeratne YD, Joshi R, Heptinstall S. Ticagrelor: a P2Y12 antagonist for use in acute coronary syndromes. Expert Rev Clin Pharmacol. 2012;5(3): 257-69. https://doi.org/10.1586/ecp.12.17.

7. Bundhun PK, Shi JX, Huang F. Head to head comparison of Prasugrel versus Ticagrelor in patients with acute coronary syndrome: a systematic review and meta-analysis of randomized trials. BMC Pharmacol Toxicol. 2017;18(1):80. https://doi.org/10. 1186/s40360-017-0189-7.

8. Norgard NB, Dinicolantonio JJ. Clopidogrel, prasugrel, or ticagrelor? a practical guide to use of antiplatelet agents in patients with acute coronary syndromes. Postgrad Med. 2013;125(4):91-102. https://doi.org/10.3810/pgm.2013.07.2682.

9. Gunarathne A, Hussain S, Gershlick AH. Prasugrel hydrochloride for the treatment of acute coronary syndrome patients. Expert Rev Cardiovasc Ther. 2016;14(11):1215-26. https://doi.org/10.1517/ 14656566.2015.1005602.

10. Bonhomme F, Fontana P, Reny JL. How to manage prasugrel and ticagrelor in daily practice. Eur J
Intern Med. 2014;25(3):213-20. https://doi.org/10. 1016/j.ejim.2014.01.016.

11. Wallentin L, Becker RC, Budaj A, et al. Ticagrelor versus clopidogrel in patients with acute coronary syndromes. N Engl J Med. 2009;361(11):1045-57. https://doi.org/10.1056/NEJMoa0904327.

12. Wiviott SD, Braunwald E, McCabe $\mathrm{CH}$, et al. Prasugrel versus clopidogrel in patients with acute coronary syndromes. N Engl J Med. 2007;357(20): 2001-15. https://doi.org/10.1056/NEJMoa0706482.

13. Kang J, Kim HS. The evolving concept of dual antiplatelet therapy after percutaneous coronary intervention: focus on unique feature of East Asian and "Asian paradox." Korean Circ J. 2018;48(7): 537-51. https://doi.org/10.4070/kcj.2018.0166.

14. Jeong YH. "East Asian paradox": challenge for the current antiplatelet strategy of "one-guideline-fitsall races" in acute coronary syndrome. Curr Cardiol Rep. 2014;16(5):485. https://doi.org/10.1007/ s11886-014-0485-4.

15. Becker RC, Bassand JP, Budaj A, et al. Bleeding complications with the P2Y12 receptor antagonists clopidogrel and ticagrelor in the PLATelet inhibition and patient Outcomes (PLATO) trial. Eur Heart J. 2011;32(23):2933-44. https://doi.org/10.1093/ eurheartj/ehr422.

16. Goto S, Huang CH, Park SJ, Emanuelsson H, Kimura T. Ticagrelor vs. clopidogrel in Japanese, Korean and Taiwanese patients with acute coronary syndrome-randomized, double-blind, phase III PHILO study. Circ J. 2015;79(11):2452-60. https://doi.org/ 10.1253/circj.CJ-15-0112.

17. Sheikh RS, Geroldinger A, Heinze G, Reichardt B, Wolzt M. Clopidogrel, prasugrel, or ticagrelor use and clinical outcome in patients with acute coronary syndrome: a nationwide long-term registry analysis from 2009 to 2014. Int J Cardiol. 2017;235: 61-6. https://doi.org/10.1016/j.ijcard.2017.02.096.

18. Kim C, Shin DH, Hong SJ, et al. One-year clinical outcomes of ticagrelor compared with clopidogrel after percutaneous coronary intervention in patients with acute myocardial infarction: from Korean Health Insurance Review and Assessment Data. J Cardiol. 2019;73(3):191-7. https://doi.org/ 10.1016/j.jjcc.2018.08.005.

19. Park KH, Jeong $\mathrm{MH}, \mathrm{Ahn} \mathrm{Y}$, et al. Comparison of short-term clinical outcomes between ticagrelor versus clopidogrel in patients with acute myocardial infarction undergoing successful revascularization; from Korea Acute Myocardial Infarction Registry-National Institute of Health. Int J Cardiol. 2016;215:193-200. https://doi.org/10.1016/j.ijcard. 2016.04.044. 
20. Kim JA, Yoon S, Kim LY, Kim DS. Towards actualizing the value potential of Korea Health Insurance Review and Assessment (HIRA) data as a resource for health research: strengths, limitations, applications, and strategies for optimal use of HIRA data. J Korean Med Sci. 2017;32(5):718-28. https://doi. org/10.3346/jkms.2017.32.5.718.

21. Mehran R, Rao SV, Bhatt DL, et al. Standardized bleeding definitions for cardiovascular clinical trials: a consensus report from the Bleeding Academic Research Consortium. Circulation. 2011;123(23): 2736-47. https://doi.org/10.1161/ CIRCULATIONAHA.110.009449.

22. Sikka R, Xia F, Aubert RE. Estimating medication persistency using administrative claims data. Am J Manag Care. 2005;11(7):449-57.

23. Blin P, Dureau-Pournin C, Benichou J, et al. Secondary prevention of acute coronary events with antiplatelet agents (SPACE-AA): one-year real-world effectiveness and safety cohort study in the French nationwide claims database. Atherosclerosis. 2019;281:98-106. https://doi.org/10.1016/j. atherosclerosis.2018.11.037.

24. Park DW, Kown OS, Jang JS, et al. Clinically significant bleeding with ticagrelor versus clopidogrel in Korean patients with acute coronary syndromes intended for invasive management: a randomized clinical trial. Circulation. 2019. https://doi.org/10. 1161/CIRCULATIONAHA.119.041766 ((Epub ahead of print)).

25. Levine GN, Jeong YH, Goto S, et al. World Heart Federation expert consensus statement on antiplatelet therapy in East Asian patients with ACS or undergoing PCI. Glob Heart. 2014;9(4):457-67. https://doi.org/10.1038/nrcardio.2014.104.

26. Park JB. Treatment guideline for Acute Coronary Syndrome. Abstr 64th Fall Conf Korean Assoc Intern Med. 2013;83:235-9.

27. The reimbursement guideline for anti-platelet treatment. Korean Insurance Change Review Association. https://www.hicra.or.kr/sub_asp/04_ data01.html?mode=read\&read_no=596\&now_ page $=1$

28. Grines C, Bonow RO, Casey DE Jr, et al. Prevention of premature discontinuation of dual antiplatelet therapy in patients with coronary artery stents: a science advisory from the American Heart Association, American College of Cardiology, Society for Cardiovascular Angiography and Interventions, American College of Surgeons, and American Dental Association, with representation from the American College of Physicians. Circulation. 2007;115:813-8. https://doi.org/10.1016/j.jacc. 2007.01.003.

29. Kim J. Strategies to enhance the use of National Health Insurance claims database in generating health statistics. Seoul: Health Insurance Review Assessment Services; 2005.

30. Park B, Sung J, Park K, Seo S, Kim S. Strategies to improve the validity of diagnostic codes of National Health Insurance claims data. Seoul: Health Insurance Review Assessment Services; 2002.

31. Park JK, Kim KS, Kim CB, et al. The accuracy of ICD codes for cerebrovascular diseases in medical insurance claims. Korean J Prev Med. 2000;33(1):12.

32. Kim MH, Yun JE, Lee SH, Jang Y, Jee SH. 2012) Validity of the diagnosis of acute myocardial infarction in Korean national medical health insurance claims data: the Korean Heart Study (1). Korean Circ J. 2012;42(1):10-5. https://doi.org/10. 4070/kcj.2012.42.1.10.

33. Yun JE, Kim YJ, Park JJ, Kim S, Park K, Cho MS, Nam GB, Park DW. Safety and effectiveness of contemporary P2Y12 inhibitors in an East Asian population with acute coronary syndrome: a nationwide population-based cohort study. J Am Heart Assoc. 2019;8(14):e012078. https://doi.org/ 10.1161/JAHA.119.012078.

34. Ooba N, Setoguchi S, Ando T, et al. Claims-based definition of death in Japanese claims database: validity and implications. PLoS ONE. 2013;8(5): e66116. https://doi.org/10.1371/journal.pone. 0066116 